\title{
Medium und Grenze: \\ Der Leib als Kategorie der Intersubjektivität. Phänomenologie und Anthropologie im Dialog
}

\section{Einleitung}

In der Phänomenologie nimmt der Leib von jeher eine zentrale Stellung in der Analyse der Intersubjektivität ein. Die Bühne der Phänomenologie betritt der Leib dabei zum ersten Mal in Edmund Husserls Analysen in den Ideen II. Zur gleichen Zeit, da die transzendentale Wende und die Ausgestaltung der phänomenologischen Reduktion Gestalt annimmt, erscheint der Leib in Husserls Texten zunehmend in der Rolle des Vermittlers zwischen Natur und Geist, sowie dem eigenen und dem fremdem Bewusstsein.

Die vermittelnde Funktion des Leibes setzt sich in der Phänomenologie von Maurice Merleau-Ponty unter nicht-transzendentalen Vorzeichen in der Beschreibung des leiblichen Ausdrucks und der Zwischenleiblichkeit fort. In beiden Ansätzen stellt der Leib unser Medium zur Welt und den Anderen dar, wenn auch mit unterschiedlichen Gewichtungen. Zugleich stellt der Leibkörper in Bezug auf die Welt und die Anderen eine Grenze dar, die die Selbstwahrnehmung wie auch die Möglichkeiten der Einfühlung in andere Subjekte empfindlich einschränkt. Der Leib ist damit nicht nur als ein ausführender Arm des Bewusstseins anzusehen - wie dies etwa der frühe Husserl suggerieren mag -, sondern konfrontiert uns in seiner Körperlichkeit, Endlichkeit und Geschichtlichkeit mit der Anonymität unseres Menschseins - was insbesondere bei Merleau-Ponty im Zentrum seiner phänomenologischen Überlegungen steht.

Der Aspekt der Grenze, der in der phänomenologischen Untersuchung meist lediglich in Gestalt einer Limitation von Wahrnehmung und Erkenntnis erscheint, stellt in der philosophischen Anthropologie hingegen - hier exemplifiziert an Helmuth Plessner - den Kern der Bestimmung der Lebewesen, insbesondere des Menschen, dar: Anthropologisch zeichnet sich der Mensch gerade durch seine Distanz zu sich und den Anderen aus. ${ }^{1}$ Die hier zugrundegelegte 
zu sich und den Anderen aus. ${ }^{1}$ Die hier zugrundegelegte Vermitteltheit des Weltbezuges ergibt sich aus der Lebensform der Positionalität, die sowohl Tieren als auch Menschen zukommt. Als solche sind wir nicht nur ein Gesamtorganismus, ein Leib, sondern sind zugleich in diesen als Zentrum gesetzt: Wir sind Leib und haben diesen als Körper. Diese Abgehobenheit vom eigenen Leib ermöglicht es, dass wir uns zu uns selbst und unserer Umwelt in Beziehung setzen können. Im Gegensatz zum Tier ist uns diese Vermitteltheit zwischen Innen und Außen jedoch bewusst, wir nehmen zusätzlich einen exzentrischen Standpunkt ein (Plessner 1965, 325). Durch unsere Körperlichkeit sind wir insofern nicht nur räumlich nach Innen und Außen begrenzt, sondern müssen diese Grenze vielmehr beständig neu bestimmen und realisieren.

Was also macht den Leib sowohl zum Medium als auch zur Grenze unseres Selbst- und Weltbezuges? Wie hängt die phänomenologisch unmittelbare Zwischenleiblichkeit mit dem Aspekt der exzentrischen Positionalität zusammen? Um diesen Fragen näher zu kommen, sollen hier die Phänomenologie Husserls und Merleau-Pontys in einen Dialog mit der philosophischen Anthropologie Plessners gebracht werden. Steht in der Tradition der Phänomenologie der subjektive Leib als Medium im Vordergrund, kann die anthropologische Perspektive Aufschluss geben über das spezifische Körperhaben des Menschen und seine begrenzende Funktion aus einer methodisch eingenommenen Außenperspektive.

Eine solche ,Außenperspektive' oder aber ,geschichtlichintersubjektive' Perspektive auf den Körper tritt ebenfalls in der Wahrnehmung und Bewertung von eigenem und fremdem Körper in Kraft - allerdings nicht in Form einer beabsichtigten, methodisch eingenommenen Sichtweise wie etwa bei Plessner, sondern auf implizite Weise. Um diese kulturell-geschichtliche Diskursivität unserer Körperwahrnehmung und ihr Zusammenspiel mit unserem operativ

Plessner stellt seinen methodischen Ansatz in Abgrenzung zu Heideggers Fundamentalontologie dar. Gleichzeitig soll er die phänomenologischen Untersuchungen anthropologisch fundieren. Die Anthropologie soll insofern die Entwicklungsgeschichte des Bewusstseins aufzeigen, die nicht erst mit dem Menschen beginnt. Dabei muss sich die Untersuchung rein auf die, äußerliche Anschauung' beschränken und von einem Faktum ausgehen: Als Faktum, das allen Lebewesen gemein ist, setzt Plessner die Abgrenzung/Begrenzung gegen eine Umwelt. Diese, Grenze gilt als Minimalbedingung des Lebendigen. Vgl. Plessner 1965, XIV-XXI. Ähnliche Kriterien zur Definition von Leben verwenden die Vertreter des AutopoiesisAnsatzes, allerdings ohne auf Plessner Bezug zu nehmen. Vgl. Maturana \& Varela 1980; Varela, Thompson \& Rosch 1992; Thompson 2007. 
wirksamen Leibsein in den Blick zu bekommen, soll daher abschließend für eine Zusammenarbeit von Phänomenologie, Anthropologie und den kulturgeschichtlichen Disziplinen der Medizin- und Körpergeschichte plädiert werden. Der Leib erscheint somit nicht nur als Medium und Grenze konkreter intersubjektiver Begegnung, sondern zugleich als Subjekt und Objekt kultureller und normativer Konstitution des Bildes vom normalen oder pathologischen, gesunden oder kranken sowie dem schönen oder hässlichen Körper.

\subsection{Das methodische Gerüst der Beschreibung}

Um den Leib als Kategorie der Intersubjektivität unter diesen Vorzeichen systematisch beschreiben zu können, sollen im Folgenden zwei Untersuchungsebenen unterschieden werden. Die vertikale und die horizontale Untersuchungsebene. Während die horizontale Untersuchungsebene das Leibsein beinhaltet, entspricht die vertikale Perspektive dem Körperhaben (vgl. ebd., 31f.). Das Leibsein zählt somit zum Aufgabengebiet der Phänomenologie (hier: insbesondere MerleauPontys Leibtheorie). Während sich die horizontale Untersuchung den Formen des leiblichen Zur-Welt-seins widmet, beschäftigt sich die vertikale Untersuchungsebene (Anthropologie) mit der Stellung des Menschen im Vergleich zu anderen Formen des Lebendigen. Hieran knüpfe ich für die weiteren Überlegungen folgende Arbeitshypothesen:

1. Leibsein und Körperhaben entsprechen verschiedenen Stufen der Intersubjektivität.

2. Eine umfassende Untersuchung der Intersubjektivität verlangt eine Verbindung von phänomenologischer, anthropologischer Perspektive und kulturwissenschaftlicher Perspektive: also der subjektiven Perspektive des Leibseins (ErstePerson-Perspektive), der vergleichenden Perspektive der Anthropologie und der geschichtlich-diskursiven Perspektive der Kulturwissenschaften, die Bewertungsschemen unserer intersubjektiv geprägten Körperwahrnehmung sichtbar macht.

Die schematische Differenzierung in eine vertikale und horizontale Untersuchungsebene soll hierbei lediglich als Orientierung dienen. 
Selbstverständlich lassen sich die beschriebenen Sachverhalte in ihrer Komplexität nicht vollständig durch eine solche Schematisierung abbilden. Im ,Menschsein' kommt es notwendigerweise zu Überschneidungen der Inhalte von Leibsein und Körperhaben. Weiterhin sind die dargestellten Positionen von Husserl, Merleau-Ponty und Plessner nicht eins zu eins den obigen Untersuchungsebenen zuzuordnen. Nichtsdestotrotz hält die Autorin eine solche schematische Unterscheidung für sinnvoll, da sie die Diskrepanz zwischen den beiden Beschreibungsebenen aufzeigt, deren eine Seite von der Phänomenologie und deren andere Seite von Anthropologie und Kulturwissenschaft vernachlässigt werden. Die Notwendigkeit eines Dialoges der Disziplinen, der zur Aufklärung der Rolle der Leiblichkeit und Körperlichkeit des Menschen als Voraussetzung und Charakterisierung seiner intersubjektiven Beziehungen und Abhängigkeiten dienen soll, wird hierdurch ersichtlich.

\section{Husserl: Der Leib und die Intersubjektivität}

„Derselbe Leib, der mir als Mittel aller Wahrnehmung dient, steht mir bei der Wahrnehmung seiner selbst im Wege und ist ein merkwürdig unvollkommen konstituiertes Ding." (Hua IV, 158) Dieses allseits bekannte Zitat aus den Ideen II vereint sehr eindrücklich die beiden Aspekte, die für Husserl die Beschreibung des Leibes wesentlich ausmachen und die in seiner Nachfolge die weitere Bestimmung dieses Phänomens prägen sollten. Einerseits zeichnet sich der Leib durch seine vermittelnde Funktion aus, die uns das Wahrnehmen überhaupt erst ermöglichen. Er stellt sich somit als „Medium unseres Bezuges zur Welt“ (Waldenfels 2000, 210) dar und wird als Bindeglied zwischen Geist und Natur, sowie als ,Umschlagstelle' zwischen Innen und Außen, Subjekt und Welt definiert. Andererseits wird er als hinderliche Instanz charakterisiert, die uns zu unserer eigenen körperlichen Erscheinung nur beschränkten Zugang ermöglicht. Dass ich selbst dieser Leib bin und er somit stets bei mir und ständig für mich da ist, „besagt in eins, dass ich [ihn] niemals [...] eigentlich vor mir habe" (Merleau-Ponty 1966, 115).

Den Leib kann Husserl also nicht wie einen herkömmlichen Gegenstand des Bewusstseins behandeln. Als Empfindungsfeld für die Bewusstseinsinhalte und aufgrund seiner kinästhetischen Funktionen muss der Leib selbst als konstituierend gelten, wie sich in den späte- 
ren Schriften Husserls mehr und mehr zeigt. ${ }^{2}$ Die eigentümliche Spaltung des Leibes in ein subjektives und objektives, Gesicht' macht Husserl am berühmten Beispiel der Doppelempfindung deutlich: Wenn die linke Hand die rechte berührt, ist der Leib einerseits Instanz der Wahrnehmung und andererseits Wahrnehmungsobjekt. Wird die betastete Hand als Ding wahrgenommen, also hinsichtlich ihrer physischen Merkmale, so kann dies nur durch ,Abstraktion ihrer ebenso lokalisierten Empfindungen geschehen. Sobald man diese aber hinzunimmt, wird es "Leib, es empfindet“ (Hua IV, 145).

Diese „doppelte Konstitution des Leibes“ (ebd.) ähnelt dem Phänomen und der Begrifflichkeit nach dem, was Plessner unter „Doppelaspektivität" (Plessner 1965, 70) versteht. Mit Doppelaspektivität meint Plessner die Wesenseigenschaft jedes Lebendigen, zwei Richtungen aufzuweisen: Innen und Außen. Dieses Auseinanderfallen in ein Innen und ein Außen weist bereits der nicht-organische Körper auf, seine äußere Gestalt verweist auf ein Innen. Weist das nichtlebendige Ding als Grenze nur einen äußerlichen Rand auf, mit dem es an andere Dinge stößt, erscheint die Doppelaspektivität beim lebendigen Organismus als anschauliche Vermittlung der beiden Sphären durch eine Grenze, die selbst Eigenschaft dieses Körpers ist. Diese Grenze muss sowohl Raumgrenze als auch Aspektgrenze sein, in welcher der Umschlag zweier wesensmäßig ineinander nicht überführbarer Richtungen stattfindet (vgl. ebd., 100f.). Das heißt, der aus der Dritten-Person-Perspektive der Anthropologie beobachtete Körper gilt dann als lebendig, wenn sein Außen auf eine innere Organisation verweist. Als Beispiel für eine solche innere Organisation kann etwa der leibliche Ausdruck genannt werden. Das Lebendige zeichnet sich bei Plessner insofern allgemein durch den Bruch zweier Sphären aus, deren Einheit beständig einer dialektischen Vermittlung durch die Grenze geleistet werden muss. Die Stufen des Organischen, von der Pflanze zum Tier bis hin zum Menschen, unterscheiden sich demnach dadurch, wie diese Grenze im Einzelnen realisiert wird. Dies führt von der offenen Positionalität der Pflanze, die in ihre Umwelt eingebettet ist, zur geschlossenen Positionalität des Tieres, die sich durch die Selbstständigkeit und Abgeschlossenheit gegenüber der Umwelt und eine zentrale Organisation auszeichnet. In der geschlossenen Positionalität tritt nun eine Spaltung von Leibsein und Körperhaben auf, da das Tier seine Bewegungen zentral kontrollieren kann.

2 So etwa in den späteren Forschungstexten zur Intersubjektivität (Hua XIII-XV). 
Es ist damit sein Körper, gleichzeitig ist es aber auch als Ausführender in diesen Körper gesetzt. ${ }^{3}$ Dies ermöglicht nach Plessner eine Gegenüberstellung zur Umwelt und damit die Möglichkeit eines Verhaltens zur Umwelt, zu Anderen und zu sich selbst. Diese Vermitteltheit ist dem Tier jedoch nicht bewusst. Erst der Mensch hat eine Einsicht in die vermittelte Unmittelbarkeit seines Selbst- und Weltverhältnisses. Dies macht seine exzentrische Position aus.

Husserls Begriff der doppelten Konstitution beschreibt ein ähnliches Phänomen, er stellt jedoch keine anthropologische Feststellung dar. Ob der Leib als Subjekt oder Objekt betrachtet wird, hängt von der Einstellung des Bewusstseins, der jeweiligen Aufmerksamkeit ab. Die transzendentale Blickrichtung bei Husserl offenbart dabei in gleicher Weise wie die ,objektive' Beobachtung bei Plessner eine dreifache Positionalität. Aus der Perspektive des konstituierenden Bewusstseins stellt der Leib das Medium bzw. den Gegenstand des Geistes dar, während wiederum der Leibkörper für die verweltlichte Subjektivität (die Person) als Werkzeug und Objekt fungieren kann. Sowohl die Verweltlichung des transzendentalen Subjekts als leibliche Person als auch ihr Leibkörper stellen dabei den Ausgangspunkt der konkreten Fremderfahrung bei Husserl dar. ${ }^{4}$

Ihren Anfang nimmt die Fremderfahrung oder Einfühlung in der Wahrnehmung des sichtbaren Leibkörpers des Anderen, hier findet zunächst eine assoziative Sinnübertragung vom Original (meinem Leib) auf den fremden Körper statt. Beide Körper werden aufgrund ihrer Ähnlichkeit strukturell als zusammengehörig, d.h. als Paar aufgefasst (vgl. Hua I, 141f.). Auf der Basis dieser Paarung durch Ähnlichkeit bauen sich nun höherstufige Einfühlungen auf, in welchen ich dem gesehenen Körper nicht nur meine leiblichen Eigenschaften, sondern auch meine geistigen Fähigkeiten übertrage. Hierzu muss ich mich aber in die Lage und Bewegungen des Anderen regelrecht hineinversetzen, dies kann ich nur aufgrund der kinästhetischen Funktionen meines Leibes. Ich sehe damit den Körper des Anderen, als wenn ich dort wäre. Beschrieb Husserl diese Einfühlungsprozesse in früheren Schriften mit mentalen Begrifflichkeiten, sodass der Ein-

3 Plessner spricht hier nicht von einem Leibsein, sondern nur von dem Körper oder dem Gesamtorganismus, der man ist, im Gegensatz zu dem Körper, den man hat. Um einen Dialog zwischen Phänomenologie und philosophischer Anthropologie zu erleichtern bzw. die Begrifflichkeiten einander anzunähern, wird die Ebene des Körperseins hier als Leibsein im Sinne einer subjektiv fungierenden Leiblichkeit bezeichnet.

4 Für eine genauere Darstellung siehe Wehrle (2010). 
druck entstand, es handele sich um eine gedankliche Einfühlung oder einen Analogieschluss, macht er in späteren Texten deutlich, dass die Einfühlung ihren Ursprung in einer passiv-genetischen Leistung des Bewusstseins bzw. des Leibes nimmt. Er spricht diesbezüglich sogar von einer wechselseitigen Sinnangleichung und Verähnlichung der Leiber (vgl. ebd., 147), die während dieses Prozesses stattfindet. Dies erinnert stark an dasjenige, was Merleau-Ponty später - unter nichttranszendentalen Vorzeichen - mit dem Begriff der Zwischenleiblichkeit bezeichnet.

Der Leib gilt somit einerseits als Mittel bzw. Medium der Intersubjektivität, indem er dem Geist konkret Ausdruck verleiht: Er vermittelt in der "faktischen Welt die Verständigung der Geister" und "der ganzen Menschen nach ihrem Seelenleben" (Hua XIII, 230). Andererseits wird die Körperlichkeit als Grenze betrachtet, die uns den Eingang ins Innenleben des Anderen geradezu verwehrt. Die Intersubjektivität ist damit definiert als die „bewährbare Zugänglichkeit eines original Unzugänglichen" (Hua I, 144). Die Voraussetzung für den intersubjektiven Kontakt bildet dabei mein Eigenleib mit seinen Empfindungen und Wahrnehmungen. Doch ist es gerade die Spaltung in Leib und Leibkörper, die Selbstobjektivierung, die es uns möglich macht, andere als Leiber und Subjekte wahrzunehmen und uns selbst mit den Augen eines Anderen zu sehen. Diese Selbstobjektivierung ist nach Husserl bereits im Leib bzw. im Bewusstsein vom Leib selbst angelegt und wird nicht etwa wie bei Sartre erst durch den Blick des Anderen hervorgerufen (Sartre 1993, 457ff.).

Aus der anthropologischen Perspektive ist es ebenfalls der Aspekt der Distanz bzw. der Vermittlung, der Intersubjektivität erst ermöglicht. Hier findet allerdings keine Selbstobjektivierung statt, es besteht kein Vorrang des leiblichen Selbstbezuges vor dem Körperhaben. Aus der Außenperspektive der Anthropologie erweisen sich Leibsein und Körperhaben als gleichursprünglich. In seinem Leibsein als Positionalität ist es dem Menschen daher genau wie den Tieren möglich, zu anderen Menschen und Leibern in Beziehung zu stehen. Dies würde der Ebene des leiblichen Ausdrucks und der Zwischenleiblichkeit entsprechen, welche Merleau-Ponty in seiner Leibtheorie erläutert.

Die Einsicht des Menschen in die Mittelbarkeit seines Selbst- und Weltbezuges, also darin, dass er einen Körper hat, ermöglicht es ihm darüber hinaus, sich explizit in die Position des Anderen hineinzuversetzen. Die Ebene des Körperhabens gilt somit als Voraussetzung für höhere, d.h. explizitere Formen der Intersubjektivität und Sozialität. 
Die exzentrische Positionalität verhindert damit nicht nur, dass der Mensch in seinem Leibsein aufgehen kann, sondern zwingt ihn zugleich, einen utopischen Standpunkt einzunehmen: Der Mensch sieht sich und seinen Körper immer im Vergleich zu Anderen, er bewertet den eigenen und fremden Körper und wird wiederum von Anderen bewertet. Die Art und Weise, wie wir unseren Körper sehen und bewerten - das Körperhaben bzw. unser Körperbild - hängt somit ganz maßgeblich von den Blicken der Anderen im Sinne Sartres ab, und damit verbunden von den kulturellen Diskursen und Maßstäben, die diese Blicke repräsentieren. So steht der Leib uns als , unvollständig konstituiertes Ding', als Körper, den wir haben, bei seiner eigenen Wahrnehmung auch deshalb im Wege, da diese bereits intersubjektive Normen und Diskurse miteinschließt. Gleichzeitig fungiert er als Subjekt, als Leibsein, selbst konstituierend in Bezug auf seine Umwelt und andere Leiber.

Merleau-Ponty wird dieser Linie folgend, den Leib nicht mehr als Werkzeug des Geistes oder Konstitutionsphänomen des Bewusstseins behandeln, sondern ihm selbst den Status als sinnstiftendes Wahrnehmungssubjekt verleihen. Hierbei wird aber deutlich, dass dieses seltsam unvollkommen konstituierte Ding Leib sich nicht nur als Gegenstand unserer Bestimmung entzieht, sondern uns gerade auch als Wahrnehmungssubjekt mit unserer Anonymität konfrontiert.

\section{Merleau-Ponty:}

Die Anonymität des Leibseins und die Zwischenleiblichkeit

Merleau-Ponty stellt nun den Leib nicht mehr als Vermittler zwischen Geist, Seele und Natur dar, sondern setzt ihn in ein drittes Milieu, die Existenz. Nicht mehr das Bewusstsein, das ,ich denke', wird erkenntnistheoretisch als vorgängig betrachtet, sondern die Welt, als Situierung unserer leiblichen Existenz: „Die Welt ist da, vor jeder Analyse“ (Merleau-Ponty 1966, 6). In und zu dieser Welt verhalten wir uns als faktisches leibliches Subjekt. Die Faktizität, unser Leibsein, stellt damit die Voraussetzung für jede nachträgliche Form der Reflexion dar.

Wie schon herausgestellt wurde, bestimmt Plessner dieses Leibsein anthropologisch als zentrische Positionalität, welches die Lebensformen Tier und Mensch beschreibt. Während Plessner die Distanz zu sich, der Umwelt und den Anderen als Voraussetzung eines Bezuges, 
d.h. einer Gegenüberstellung von Organismus und Umwelt definiert, betont Merleau-Ponty hingegen gerade den Aspekt der Unmittelbarkeit. Dies liegt an den unterschiedlichen Perspektiven, die ich oben als horizontale und vertikale Untersuchungsebenen bestimmt habe. Will Merleau-Ponty den Leib gegenüber einem Intellektualismus und Sensualismus als präreflexiven, ganzheitlichen Weltbezug etablieren, gilt Plessner die Ausbildung einer Grenze und Abgeschlossenheit gegenüber der Umwelt als Unterscheidungsmerkmal zwischen Pflanzen und höheren Organismen. Einmal ist es die Grenze des Organismus, die Innen und Außen vermittelt und ein anderes Mal ist der empfindende und wahrnehmende Leib selbst Subjekt und Medium. Die vertikale Untersuchungsrichtung wird dabei vom Konzept des Lebens geleitet: Sie bemüht sich, interne Spezifizierungen und Unterschiede der Lebensformen darzustellen. Bei der horizontalen Untersuchungsrichtung, für die Merleau-Ponty hier stehen soll, steht hingegen der Existenzbegriff im Vordergrund: Hier soll gerade die Verbindung und Kontinuität von Sinnlichem, Geistigem, Leib, Person und Denken in Erscheinung treten.

Was dieses Leibsein, das Plessner rein anthropologisch als positionale Lebensform festschreibt, phänomenologisch ausmacht, wird von Merleau-Ponty recht eindrücklich charakterisiert. Das Leibsein ist auf der einen Seite durch das Zur-Welt-sein, die aktuelle Interaktion und das Engagement in der Welt gekennzeichnet. Auf der anderen Seite ist der Leib in der Welt, also räumlich, zeitlich und damit auch geschichtlich situiert. Der Leib steht dabei sowohl in einem allgemeinen Horizont (biologisch, geschichtlich, intersubjektiv) als auch in einem individuellen Horizont, der seine bisherige Erfahrungsgeschichte beinhaltet. Aufgrund seiner Zeitlichkeit erweist sich das Leibsein selbst als doppeldeutig: Merleau-Ponty bezeichnet dies als die Ambiguität des Leibes. ${ }^{5}$ Er ist sowohl aktueller Leib, der in seinem ZurWelt-sein über sich hinaus weist, als auch habitueller Leib, d.h. Träger einer "sedimentierten Geschichte“ aus "unexplizit gebliebene[n] Erfahrungen" (Merleau-Ponty 1966, 450). Durch die gegenwärtige leibliche Intentionalität verbindet sich diese passive Vorgeschichte des

Die Ambiguität ist dem konkreten Leibsein wesentlich, da es verschiedene Zeitebenen in sich einschließt und zugleich überbrücken muss. Die Ambiguität steht so einerseits für die vermittelnde Rolle des Leibseins. Andererseits wird die Zweideutigkeit der leiblichen Wahrnehmung im Vergleich zum sprachlichen Ausdruck und seinen kreativen Sinnverschiebungen von Kritikern wie von Merleau-Ponty selbst als eine schlechte Ambiguität bezeichnet. Vgl. Merleau-Ponty 1972, 11 sowie Waelhens 1951; Waldenfels 1985, 64; Bermes 1994, 36; Stoller 1995, 150. 
Leibes mit möglichen zukünftigen Erfahrungen in einem „intentionalen Bogen“" (ebd., 164). Als Zusammenspiel von intentionalen und habituellen Aspekten entspricht dem Leibsein ein Körperschema. Ich habe meinen Leib somit in meinem „unmittelbaren Besitz, und die Lage eines jeden meiner Glieder weiß ich durch ein allumfassendes Körperschema" (ebd., 123). Parallel zu einem solchen impliziten und praktischen Wissen um die Stellung und das motorische Können des Leibes, lässt sich nach Thomas Fuchs ein leibliches Gedächtnis festmachen: Dieses operativ wirksame, aber nicht-thematische Gedächtnis umfasst sowohl habituelle Bewegungsabläufe, erlernte Fertigkeiten (Laufen, Fußballspielen), leiblich-emotionale Erfahrungseindrücke (traumatische Erlebnisse, Schmerzen und Wohlsein) als auch intersubjektive Beziehungsmuster und inkorporierte kulturelle Konventionen (vgl. Fuchs 2000a, 2000b, 2008). Insbesondere dem prozeduralen Aspekt eines solchen Gedächtnisses widmet sich vermehrt die Neurowissenschaft. Das sogenannte skill oder habit memory wird in entsprechenden Untersuchungen im striatalen Gedächtnissystem verortet. $^{6}$

Körperschema und leibliches Gedächtnis strukturieren unser Umfeld in bekannte und unbekannte Bereiche, gefährliche und harmlose Situationen oder interessante und uninteressante Dinge. Sie ermöglichen damit eine unmittelbare Orientierung und leiten jede weitere Erfahrung. Dem Leibsein eines jeden Subjekts kommt demnach je nach seinen Erfahrungen und Fertigkeiten (skills) ein individueller Wahrnehmungsstil zu, der sich in einer spezifisch leiblichen Aufmerksamkeit ${ }^{7}$ zeigt.

Die Ebene des Leibseins zeichnet sich somit durch eine unmittelbare sinnliche Interaktion mit und Engagement in der Welt aus, das

Es wird in diesem Zusammenhang etwa zwischen einem sequentiellen und einem habituellen Gedächtnis unterschieden (vgl. Ennen 2003).

Eine spezifisch leibliche Aufmerksamkeit wird insbesondere bei körperlich orientierten Handlungen sichtbar, wie etwa dem Gehen, Fußballspielen, Tanzen etc. In diesem Zusammenhang lässt sich zwischen erlernten und erworbenen leiblichen Fertigkeiten sowie zwischen Bewegungsverläufen und typischen habituellen Reaktionsformen unterscheiden. Die leibliche Aufmerksamkeit ist dem ausführenden Subjekt zwar nicht in derselben Form thematisch wie höhere kognitive Aufmerksamkeitsprozesse, lässt sich jedoch durch eine ausgeprägte Wachsamkeit gegenüber sämtlichen Umweltvorgängen charakterisieren. Sie zeichnet sich insofern nicht durch eine detaillierte fokale Wahrnehmung, sondern durch ihren situativen Charakter aus. Leibliche Aufmerksamkeit zeigt sich etwa in der körperlichen Spannung, Ausrichtung und Wachsamkeit in Bezug auf die umgebenden Ereignisse, die zum Beispiel das Verhalten eines Fußballspielers ausmachen. 
zeitliche und habituelle Ebenen integriert: Sie ist passiv-assoziativ, präreflexiv, nicht-thematisch, fungiert automatisch und bezieht sich dabei nicht auf vereinzelte Gegenstände, sondern auf Situationen und gestalthafte bzw. vor-gegenständliche Einheiten. Parallel zu diesem permanenten Weltbezug weist sie einen unmittelbaren Selbstbezug in der Empfindung auf (vgl. Hua III/1, 153ff.; Zahavi 2003, 105). Dieses selbstverständliche Fungieren der leiblichen Ebene wird erst dann eigentlich thematisch, wenn es zu Unterbrechungen und Störungen kommt. Beispiele hierfür können zur Genüge im Alltag gefunden werden, wie etwa das Stolpern über ein Hindernis während eines Spazierganges, das die bis dahin fungierende Bewegungsroutine jäh unterbricht. Neben diesen kleinen Unterbrechungen des Alltags zeichnen sich insbesondere psychische Pathologien durch einen beständigen Verlust der Selbstverständlichkeit in Wahrnehmung und Handlung aus. Als Beispiel kann hier die fehlende Integration der Zeitebenen bei Schizophrenen gelten, welche eine kontinuierliche Wahrnehmung verhindert und zu einem Zustand führt, in dem Veränderungen völlig unerwartet und ohne Vorwarnung passieren und regelrecht in die Wahrnehmung des betroffenen Subjekts hereinbrechen. Thomas Fuchs sowie Shaun Gallagher erklären dieses Symptom durch ein Fehlen von Antizipation, das durch eine Störung der Protention innerhalb der Zeiterfahrung begründet ist (vgl. Fuchs 2007 sowie Gallagher 2005, 189-200). ${ }^{8}$ Ebenfalls ein Beispiel für ein solches Fehlen des automatisch fungierenden Leibseins bzw. des Körperschemas stellt der Fall von Ian Waterman dar, der an Deafferenzierung leidet. Vom Hals abwärts verfügt er über keinerlei taktuelle oder propriozeptive Empfindungen. Die fehlende Rückkopplung seiner Bewegungen durch Bewegungsempfindungen (Propriozeption) verhindert somit die automatische motorische Kontrolle seiner Glieder. Um einfache Bewegungen auszuführen, müssen diese nun von Waterman rein visuell kontrolliert werden, was jedoch langer Übung und dezidierter Konzentration bedarf (Cole 1995).

Dem oben beschriebenen fungierenden Leibsein, welches uns oft nur in seinen Unterbrechungen offenbar wird, entspricht nun eine spezielle Form der Intersubjektivität, die Merleau-Ponty als Zwischen-

8 Beide Autoren interpretieren dieses Phänomen als eine Störung der zeitlichprotentionalen Struktur der Erfahrung. Die Patienten berichten von einem ungefilterten Ausgeliefertsein in Bezug auf wechselnde äußere Eindrücke. Dies manifestiert sich als ein Fehlen von selbstbestimmten Intentionen und Handlungsinitiative (agency). 
leiblichkeit (intercorporéité) bezeichnet (vgl. Merleau-Ponty 1994, 194; 2003, 256). ${ }^{9}$ Empfindungen und Gefühle manifestieren sich hier unmittelbar im leiblichen Ausdruck, ohne einen Umweg über das vorstellende oder vermittelnde Bewusstsein nehmen zu müssen: Der Zorn sitzt regelrecht in der geballten Faust oder im erröteten Gesicht. Ich fasse den Zorn insofern nicht als einen psychischen $\mathrm{Zu}$ stand auf, der sich hinter der Geste verbirgt: Ich begegne dem Zorn direkt im leiblichen Ausdruck meines Gegenübers: Die Geste der geballten Faust lässt mich nicht etwa an Zorn denken, „sie ist der Zorn“ (Merleau-Ponty 1966, 219). Um den Anderen zu verstehen, muss ich mir demnach nicht erst meine eigenen Gefühle in Erinnerung rufen oder mich emotional an die Stelle des Anderen versetzen. Im Gegenteil: Die Gefühle des Anderen verstehe ich, sobald ich sie sehe, und stelle mich unmittelbar leiblich darauf ein: Da ich die „Mimik des Zornes nur schlecht von innen“ kenne, fehlt für „Ähnlichkeitsassoziationen oder Analogieschlüsse“ das „entscheidende Element" (ebd.).

Der Leib fungiert hier nicht mehr nur als Medium, mit dem ich zu den Dingen gelange, sondern lässt mich Andere unmittelbar verstehen: „Durch meinen Leib verstehe ich den Anderen, so wie ich auch durch meinen Leib die "Dinge“ wahrnehme." (Ebd., 220) Der Sinn liegt in beiden Fällen nicht hinter dem Sichtbaren, sondern im sinnlichen Geschehen selbst. Wenn zwei,Leiber' sich begegnen, bildet sich zwischen ihnen ein Kräftefeld der Interaktion: In diesem findet unmittelbar ein gegenseitiges Abtasten, ein wechselseitiges Aufeinanderabstimmen, eine regelrechte Synchronisation statt. Die Zwischenleiblichkeit ist somit als eine Situation charakterisiert, in die beide Partner von vorneherein einbezogen sind und dessen Sinn die einzelnen Leiber übersteigt. Diese unmittelbare Verbindung zwischen meinem Leib und anderen Leibern erweist sich in der entwicklungspsychologischen und neurowissenschaftlichen Forschung auch als biologisch fundiert. Experimente zum Imitationsverhalten bei Neugeborenen (vgl. Meltzoff \& Moore 1989; 1997; Meltzoff 2005) oder die Entdeckung von Spiegelneuronen (vgl. Rizzolatti et al. 1996; Rizzolatti \& Craighero 2004; Gallese 2003), die eine Verbindung der Verarbeitung eigener Bewegung und der Bewegungswahrnehmung anderer Menschen aufzeigen, weisen auf eine Entsprechung der intersubjekti-

9 Für eine Darstellung des Konzepts der Zwischenleiblichkeit siehe Waldenfels (2000, 287ff.), Fuchs (2003) und Fuchs \& De Jaegher (2009). 
ven Phänomene auf phänomenologischer Ebene und den empirischen Gegebenheiten der Neurophysiologie des Menschen hin. ${ }^{10}$

Im Zusammenhang mit der intersubjektiven Dimension der Zwischenleiblichkeit offenbart sich zugleich ein spezifischer Aufmerksamkeitsstil des Leibes, welcher die individuelle Erfahrungsgeschichte eines konkreten Leibes, die Erlebnisse, Gewöhnungen und erlernte Fertigkeiten mit einschließt: Neuropsychologische Studien konnten hierbei zeigen, dass das, was wir sehen, und das, worauf wir im Einzelnen aufmerksam sind, sowohl von unseren momentan ausgeführten Handlungen als auch von unseren habituellen Fähigkeiten abhängt. Bei einem Experiment mit Ballett- und Capoeiratänzern wurde die Aktivität bestimmter Gehirnregionen bei der Beobachtung der jeweils anderen Tänzer gemessen. Je nachdem, ob es sich bei der beobachteten Bewegung um die eigene Tanzdisziplin oder um ein nicht selbst erlerntes Bewegungsmuster handelte, unterschieden sich die gemessenen Aktivitäten maßgeblich. Bei der Betrachtung der eigenen Tanzdisziplin herrschte dabei eine erhöhte Aktivität prämotorischer und parietaler Gehirnregionen, was auf eine verstärkte ,motorische Aufmerksamkeit' hindeutet. Bei der Wahrnehmung von gewohnten und bekannten Bewegungen konnten die Probanden ebenfalls bessere Diskriminierungsleistungen erzielen. Bewegungsverläufe und Situationen, die mit den eigenen motorischen Handlungen und Erwerben in Verbindung stehen, gehen demnach mit einer verstärkten Aktivierung des motorischen Systems und einer differenzierteren, detaillierteren Wahrnehmung der Probanden einher. Dasjenige, so scheint es, was man durch eigene Erfahrung und Ausübung ,kennt' und das den eigenen Interessen entspricht, sieht man qualitativ anders, als herkömmliche Dinge und Situationen. Hiermit zeigt sich zugleich eine individuelle Form der Affektivität, die je nach dem jeweiligen ,Reiz und dessen Verbindung zur eigenen impliziten Erfahrungsgeschichte unterschiedlich ausfällt. Das leibliche Engagement in Bezug auf An-

10 Welcher Art diese Entsprechungen sind, ist Gegenstand zahlreicher Diskussionen: Besteht zwischen den neuronalen, physiologischen Grundlagen und den beschriebenen Phänomenen ein streng kausaler Zusammenhang, ,superveniert' etwa das Eine über das Andere? Sollen alle Formen von Empathie, Intersubjektivität und Sozialität auf solche subpersonalen, neuronalen Strukturen reduziert werden? Welche dieser Strukturen sind angeboren und welche bilden sich aufgrund von wiederholter Interaktion mit der Umwelt im Sinne der Plastizität des Gehirns? Dies sind wichtige Fragen, die man sich stellen muss, bevor man eine einfache Entsprechung oder Bestätigung von phänomenologischen Beschreibungen durch experimentelle Befunde feststellt. An dieser Stelle kann auf diese Fragen aber lediglich hingewiesen werden. 
dere und die Zwischenleiblichkeit scheint demnach nicht neutral zu sein, sondern sind besonders ausgeprägt in Bezug auf andere leibliche Subjekte, die sich in für uns bekannter Weise bewegen oder deren Bewegungen in direkter Verbindung mit unseren momentanen Handlungen stehen (vgl. Calvo-Merino et al. 2006, 2010). ${ }^{11}$

All dies weist auf eine spezifische Form der Intersubjektivität hin, die sich auf der Ebene unseres Leibseins abspielt. Aus der horizontalen Perspektive kann diese Stufe leiblicher Intersubjektivität als genetische Fundierung der personalen Subjektivität und der höheren Stufen von Intersubjektivität gelten. Auch vertikal betrachtet lässt sich diese Ebene als evolutionär früher annehmen, da zumindest viele der zentralen Merkmale auch dem Tier zugesprochen werden können. Jedoch kommen weder die anthropologisch festgestellte Positionalität noch das phänomenologische Leibsein im Leben eines erwachsenen Menschen in reiner Form vor, sondern stehen immer in Verbindung mit personalem (d.h. explizitem, thematischen und sprachlichen) Sein. Ein Zusammenfall beider Ebenen vollzieht sich höchstens in Grenzsituationen, etwa in der von Merleau-Ponty beschriebenen Situation der Gefahr: „In einer Gefahr kann es sogar geschehen, daß die menschliche Situation die biologische auslöscht, mein Leib rückhaltlos aufgeht in meinem Tun." (Merleau-Ponty 1966, 109) Doch dies sind dies seltene Augenblicke, wie Merleau-Ponty betont. Ansonsten spielen die biologisch-leibliche und die personale Ebene des Subjekts zusammen, z.B. fungiert das Leibsein bei einem Spaziergang oder bei einer Autofahrt regelrecht automatisch, was es ermöglicht sich zugleich einem interessanten Gespräch zu widmen. Das implizit verbleibende Leibsein wird dabei meist gänzlich von der persönlichen Existenz eingenommen: „zumeist verdrängt die persönliche Ebene den Organismus, ohne ihn je überwinden noch auch je auf sich selbst verzichten zu können, ohne je ihn auf sich oder sich auf ihn reduzieren zu können (ebd.). Oft ist jedoch das Leibsein der Person dabei schon zeitlich voraus: Etwa wenn das persönliche Ich noch der Trauer des verlorenen Freundes hingegeben ist, der Blick aber bereits wieder umherschweift und sich anderen Dingen zuwendet: „Indessen ich noch von Trauer erschüttert und ganz meinem Schmerz hingegeben bin, irrt doch schon wieder mein Blick vor mir her, wendet sich un-

11 Die gesteigerte Aufmerksamkeit bzw. die erhöhte Aktivierung des motorischen Zentrums zeigt sich insbesondere, wenn es sich bei der Wahrnehmung der eigenen Tanzdisziplin (z.B. Ballett) um Darstellungen seitens des eigenen Geschlechts handelt (sofern ein geschlechtsspezifisches Bewegungsrepertoire vorliegt). 
versehens irgendeinem ihn anziehenden Gegenstand zu und beginnt aufs neue sein autonomes Dasein." (Ebd.)

Leibsein und persönliche Existenz können aber auch vollständig auseinander treten: Hier widerspricht das leibliche Gebaren dem, was uns explizit ist und dem, was wir unserer Umwelt sprachlich mitteilen: Diese Diskrepanz von sprachlicher Mitteilung und leiblichem Ausdruck spielt für die Psychotherapie eine große Rolle und weist auf psychosomatische Störungen oder Verdrängung hin (vgl. Fuchs 2003). Auch hier ,weiß unser Leibsein bereits mehr als uns als Person bewusst ist: Die Anonymität des Leibseins kann daher sowohl zeitlich als auch ihrem Inhalt nach nie ganz von der Reflexion eingeholt werden.

\section{Fazit: Leibsein und Körperhaben. \\ Die Unbestimmtheit des Menschen}

Das Leibsein, wie es hier phänomenologisch in seiner unmittelbaren Selbstbezüglichkeit, Situierung und Weltbezüglichkeit beschrieben wurde, bekommt Plessner aus der Dritten-Person-Perspektive seiner anthropologischen Betrachtung nicht recht in den Blick. Anthropologisch gesehen ist das Leibsein gleichursprünglich mit dem Körperhaben und steht mit ihm in dialektischer Verbindung. Aus phänomenologischer Perspektive hat der Leib jedoch als rückbezügliches Feld von Empfindung und Kinästhese Vorrang, da es das Körperhaben im Sinne einer expliziten Wahrnehmung und Handhabung des Körpers genetisch fundiert. Über dieses Körperhaben, d.h. wie es ist, einen Körper zu haben, sagt uns die Phänomenologie allerdings erstaunlich wenig. Dabei kann das Körperhaben - im Sinne einer Wahrnehmung, Bewertung und generellen Beziehung zum eigenen Körper - als Verbindungsglied zwischen der Ebene des Leibseins und dem personalen Selbst fungieren. Für Plessner stellt die Spaltung von Leibsein (in Plessners Begrifflichkeit: das fungierende Sein des Organismus) und Körperhaben den Ursprung für so etwas wie Bewusstsein dar. Dadurch, dass ich sowohl Gesamtkörper bzw. Organismus bin, als auch in diesen als Zentrum gesetzt bin, entsteht nicht nur eine Gegenüberstellung, eine abgeschlossene Grenze zum Umfeld, sondern auch eine interne Differenzierung: Der Organismus steht in Beziehung zu sich selbst und dieses ,Selbst ${ }^{\natural}$ hat einen Körper. In diesem Haben (das auch schon beim Tier vorkommt) stecken die Wur- 
zeln des Bewusstseins, das sich beim Menschen in seinem Wissen um diese Spaltung als exzentrische Positionalität ausdrückt.

Die Ebene des Körperhabens, die bereits von Husserl betont wird, indem er den Leib als Ding besonderer Art begreift, und bei Plessner das entscheidende Merkmal zur Beschreibung der Lebensformen Tier und Mensch darstellt, nimmt in beiden Theorien zwar formal eine wichtige Position ein, bleibt aber inhaltlich unterbestimmt. Bei Merleau-Ponty lässt sich etwas mehr über die Ebene des Körperhabens herausfinden.

Er unterscheidet in diesem Zusammenhang zwischen einem Leibsein im Sinne des Körperschemas (schéma corporel) und einem Körperbild (image du corps), das ein explizites Wissen um die Stellung, Position und Statur des Körpers beinhaltet. Körperschema und Körperbild beschreibt und die damit einhergehende Unterscheidung zwischen Greifen und Zeigen gehen auf Untersuchungen und Definitionen der Neurophysiologen Kurt Goldstein und Adhemar Gelb zurück (Goldstein \& Gelb 1918). Greifen und Zeigen sind zwar im Hinblick auf ihre physiologische Ausführung identisch, nicht jedoch in Bezug auf die jeweils operative Einstellung des ausführenden Subjekts. Das Greifen beschreibt in dieser Hinsicht eine automatische bzw. unmittelbar in eine Handlung einbezogene, sog. konkrete Bewegung, im Gegensatz zu der abstrakten Bewegung des Zeigens, die nicht im Dienste einer übergreifenden Handlung, sondern gewissermaßen für sich selbst steht. Die Differenzierung dieser beiden Ebenen motorischer Intentionalität entwickeln Gelb und Goldstein anhand des bekannten und bis heute in der Neuropsychologie diskutierten Fallbeispiels, ihrem Patienten Schneider: Dieser zog sich 1915 im Ersten Weltkrieg schwere Gehirnverletzungen aufgrund von Granatsplittern zu und wurde daraufhin Patient bei Gelb und Goldstein. Neben vielen anderen Einschränkungen weist Schneider im Bereich der abstrakten Bewegungen und der Formwahrnehmung starke Defizite auf: Es gelingt ihm etwa nicht, auf Anweisung mit dem Finger auf seine Nase zu zeigen. Er greift jedoch automatisch und mühelos an seine Nase, um diese mit einem Taschentuch zu putzen, oder um eine dort sitzende Fliege mit der Hand zu verscheuchen. Das Körperschema, welche das automatische Greifen kontrolliert, scheint hier noch weitgehend intakt zu sein, während das explizite Körperbild, das für den Akt des Zeigens notwendig ist, nur eingeschränkt abrufbar ist. In diesem pathologischen Fall treten die normalerweise in der alltäglichen Erfahrung verbundenen Ebenen von Leibsein (Greifen) 
und Körperhaben (Zeigen) auseinander (vgl. Gallagher 2005, 28f., 40f., 43f., 56f). ${ }^{12}$

Diese Darstellung von Körperschema und Körperbild wird von Shaun Gallagher aufgenommen und weiterentwickelt. Als Beispiele für das ,Auseinandertreten' der Ebenen von body schema (Leibsein) und body image (Körperhaben) greift er ebenfalls auf pathologische Fälle zurück. Während der Fall Schneider sowie der ebenfalls erwähnte Fall von Deafferenzierung bei Ian Waterman zu Störungen des body schema (des fungierenden Leibseins) gezählt werden können, gehen pathologische Phänomene wie z.B. der unilateral Neglect oder das Krankheitsbild der Anorexia Nervosa mit einer Verzerrung der Wahrnehmung des eigenen Körpers einher.

Trotz solcher Ansätze und Beschreibungen von Pathologien des Körperhabens lässt sowohl die Phänomenologie als auch die philosophische Anthropologie von Plessner eine eingehende Thematisierung der alltäglichen Wahrnehmung und Bewertung des eigenen Körpers und anderer Körper mitsamt ihren normativen Konnotationen vermissen. Eine solche inhaltliche Erweiterung könnte aber ein neues Forschungsfeld eröffnen, in dem unser Verhältnis zum eigenen Körper und $\mathrm{zu}$ anderen Körpern im Zentrum steht, welches durch ein ständiges Bewerten, Vergleichen, Verändern und Normalisieren charakterisiert ist. Diese Wahrnehmungen und Bewertungen haben zwar unseren Körper zum Gegenstand, die zugrundeliegenden Motivationen sind jedoch meist nicht-thematisch und wirken rein operativ. Was wir für schön, gesund, normal etc. bei uns und anderen halten, ist insofern implizit durch kulturelle Normen und Diskurse geprägt. ${ }^{13}$

Welch große Rolle das Körperhaben im Sinne eines Körperbildes (body-image) spielt, wird insbesondere anhand von virtuellen Darstellungen des Leibes im Internet sichtbar - etwa in Form von virtuellen Stellvertretern wie Avataren, schriftlichen Darstellungen des eigenen Befindens (Beschreibungen, Emoticons) in Chats oder der Selbstdarstellung durch Fotografien in sozialen Netzwerken (Funken 2000; 2004). Im Internet als virtuellem Universalmedium wird die mediale

12 Aufgrund der Beobachtung solcher Dissoziationen darf aber nicht darauf geschlossen werden, dass die entsprechenden Ebenen (etwa eine nicht-repräsentative und eine repräsentative Ebene motorischer Intentionalität) auch im Normalfall isoliert bzw. getrennt voneinander organisiert sind. Die aufgelisteten Beispiele entsprechen speziellen pathologischen Manifestationen von Leibsein und Körperhaben, die mit einer normalen leiblichen Intentionalität nicht direkt vergleichbar sind. Vgl. zu dieser Argumentation Jensen 2009.

13 Vgl. hierzu ebenfalls die Ausführungen von Staudigl (2012). 
(also die vermittelte und vermittelnde) sowie abgrenzende Funktion des Leibes regelrecht vorgeführt. Die Diskursivität und Geschichtlichkeit der Erfahrung und des Körpers mitsamt den damit einhergehenden Stereotypen, etwa in der Geschlechtsdarstellung, welche die Postmoderne theoretisch zu bestimmen versucht hat, findet hier ihre konkrete Materialisierung und Bestätigung (Lübke 2005; Nakamura 2002). Das Leibsein zeigt sich wiederum in der Interaktion in Videospielen oder virtuellen Welten (Nardi 2010; Boellstorff 2008). Im Sinne Merleau-Pontys kann dieses virtuelle Leibsein als Erweiterung des Körperschemas gedeutet werden. Hierbei wird die Grenze des Leibseins erweitert, ohne die Bindung an seine reale Positionalität und Geschichtlichkeit zu verlieren.

Der Körper, den man hat, entpuppt sich nicht nur hier als ein geschichtliches Konstrukt, das einer Genealogie unterzogen werden muss, wie dies in den Kulturwissenschaften schon seit den 1960er Jahren versucht wird. Besonders ertragreich ist eine solche geschichtliche Reflexion auf den Körper im Kontext der Medizingeschichte. Selbst scheinbar objektive Krankheitsbilder des Körpers erweisen sich hier als geschichtlich und kulturell variabel, wie anhand der Untersuchung von Zivilisationskrankheiten wie der Melancholie, Hypochondrie oder dem bekannten Beispiel der Neurasthenie und ihrer Behandlung im Ersten Weltkrieg deutlich wird (vgl. Fischer-Homberger 1978; Roelcke 1999; Hofer 2004; 2005). Die Medizingeschichte macht anschaulich, wie die moderne Medizin und die ,Geburt der Kliniken' als Geschichte einer Objektivierung des Körpers gelesen werden können (Stoff 1999; Lorenz 2000). Verwandte Disziplinen wie die Körper- und Geschlechtergeschichte sowie die Genderstudies weisen auf die diskursive Machbarkeit von Körpern und die normativen Maßstäbe hin, die bestimmen, welche Körper in welcher Zeit überhaupt ,von Gewicht' (Butler 1993; vgl. auch Duden 1987; Laqueur 1992) sind und damit überhaupt soziale und politische Berücksichtigung finden. In all diesen Bereichen findet sich ein reiches Arsenal, um das Körperhaben in seinen allgemeinen Strukturen und zu bestimmten Zeiten und Kulturen beschreiben zu können.

Über die Charakteristiken des Leibseins in der realen wie virtuellen Welt können uns die historischen und diskursanalytischen Untersuchungen jedoch meist ebenso wenig berichten wie die Phänomenologie über die alläglichen Weisen des Körperhabens. Im Gegenteil: In den meisten kulturwissenschaftlich und/oder diskursanalytisch orientierten Studien wird das Leibsein als essentialistisches Konstrukt ange- 
sehen. Es findet sich insofern eine explizite Abneigung gegenüber phänomenologischen Beschreibungen. Beide Perspektiven könnten jedoch verbunden werden, indem man wie Plessner das Leibsein und Körperhaben als zwei Seiten desselben Themas, des ,Menschen ' auffassen würde, der diese beständig vermitteln muss und damit sowohl Medium und Grenze zugleich darstellt. Die Anthropologie Plessners macht sich dabei nicht eines un-historischen Universalismus schuldig: $\mathrm{Da}$ die Wesensbestimmung des Menschen ja gerade in seiner Unbestimmtheit liegt, d.h. in seinem Zwang sowohl sich selbst als auch seine Umwelt stets neu zu realisieren. Diese wesensmäßige Performativität des Menschen kommt dabei der postmodernen sowie der phänomenologischen Analyse sehr entgegen.

Beide Sphären, das Leibsein und das Körperhaben sind auf ihre Weise zutiefst historisch und normativ. Eine Verbindung beider Ebenen wäre auch deshalb wünschenswert, da sich die Wechselwirkungen beider Sphären nur auf diese Weise offenbaren würden. So fundiert das Leibsein zwar mit seiner impliziten Erfahrungsgeschichte die Art und Weise, wie wir einen Körper haben, diesen sehen und mit den Augen anderer bewerten. Die so passiv-assoziativ generierten Selektionsmechanismen bestimmen, wie ich meinen und andere Körper wahrnehme. Zugleich haben die sprachlichen Zuschreibungen und expliziten Bilder des Körpers Einfluss auf unser implizites Leibsein, unsere unmittelbare Empfindung, dies zeigen Beispiele aus der Medizingeschichte. Um diese Ebenen für ein umfassendes Verständnis der menschlichen Intersubjektivität in den Blick zu bekommen, bedarf es eines ständigen Wechsels unserer Perspektiven, einer von Merleau-Ponty geforderten Hyperdialektik (Merleau-Ponty 1994, 130). Die Umsetzung einer solchen Hyperdialektik sollte eigentlich kein Problem darstellen: Da in dem ständigen Wechsel der Perspektiven nach Plessner sowohl unsere Aufgabe als auch unser genuin menschliches Wesen liegt, das in der exzentrischen Positionalität beschrieben wird.

\section{Literaturverzeichnis}

Bermes, C., Maurice Merleau-Ponty zur Einführung, 2. Aufl., Hamburg 1994 Boellstorff, T., Coming of Age in Second Life. An Anthropologist Explores the Virtually Human, Princeton 2008.

Butler, J., Körper von Gewicht, Frankfurt a.M. 1993 
Calvo-Merino, B., Grézes, J., Glaser, D. E., Passingham, R. E. \& Haggard, P., Seeing or doing? Influence of visual and motor familiarity in action observation, Current Biology 16, 2006, 1905-1910.

Calvo-Merino, B., Ehrenberg, S., Leung, D. \& Haggard, P., Experts see it all: configural effects in action observation., Psychological Research 74, 2010, 400-406.

Cole, J., Pride and Daily Marathon, Cambridge, MA 1995.

Duden, B., Geschichte unter der Haut. Ein Eisenacher Arzt und seine Patientinnen um 1730, Stuttgart 1987.

Ennen, E., Phenomenological coping skills and the striatal memory system, Phenomenology and the Cognitive Sciences 2, 2003, 299-325.

Fischer-Homberger, E., Vergessene Zivilisationskrankheiten, in: A. Mercier (Hg.), Umwelt und Mensch. Körperliche und seelische Auswirkungen, Bern u.a. $1978,9-38$.

Fuchs, T., Leib, Raum, Person. Entwurf einer phänomenologischen Anthropologie, Stuttgart 2000a

- Das Gedächtnis des Leibes, Phänomenologische Forschungen 5, 2000b, 7189.

- Non-verbale Kommunikation: Phänomenologische, entwicklungspsychologische und therapeutische Aspekte, Zeitschrift für klinische Psychologie, Psychiatrie und Psychotherapie 51, 2003, 333-345.

- The temporal structure of intentionality and its disturbance in schizophrenia, Psychopathology 40, 2007, 229-235.

- Leibgedächtnis und Unbewusstes. Zur Phänomenologie der Selbstverborgenheit des Subjekts, Psycho-Logik 3, 2008, 33-50.

Fuchs, T. \& De Jaegher, H., Enactive intersubjectivity: participatory sensemaking and mutual intercorporation, Phenomenology and the Cognitive Sciences 8, 2009, 465-486.

Funken, C., Körpertext oder Textkörper, in: B. Becker \& I. Schneider (Hg.), Was vom Körper übrig bleibt. Medialität-Körperlichkeit-Identität, Frankfurt a.M. 2000, 103-129.

- Über die Wiederkehr des Körpers in der elektronischen Kommunikation, in: S. Krämer (Hg.), Performativität und Medialität, München 2004, 307322.

Gallagher, S., How the Body Shapes the Mind, Oxford 2005.

Gallese, V., The roots of empathy. The shared manifold hypothesis and the neural basis of intersubjectivity, Psychopathology 36, 2003, 171-180.

Goldstein, K. \& Gelb, A., Psychologische Analysen hirnpathologischer Fälle auf Grund von Untersuchungen Hirnverletzter, Zeitschrift für die gesamte Neurologie und Psychiatrie, 41, 1918, 1-142.

Hofer, H.-G., Nervenschwäche und Krieg. Modernitätskritik und Krisenbewältigung in der österreichischen Psychiatrie (1880-1920), Wien 2004.

- Nerven, Kultur und Geschlecht - Die Neurasthenie im Spannungsfeld zwischen Medizin- und Körpergeschichte, in: F. Stahnisch \& F. Steger (Hg.), Medizin, Geschichte und Geschlecht. Körperhistorische Rekonstruktionen von Identitäten und Differenzen, Stuttgart 2005, 225-245. 
Husserl, E. (Hua I), Cartesianische Meditationen und Pariser Vorträge, hg., von S. Strasser, Dordrecht 1950.

- (Hua III/1), Ideen zu einer reinen Phänomenologie und phänomenologischen Philosophie. Erstes Buch, hg. von K. Schuhmann, Den Haag 1977.

- (Hua IV), Ideen zu einer reinen Phänomenologie und phänomenologischen Philosophie. Zweites Buch, hg. von M. Biemel, Den Haag 1952.

- (Hua XIII), Zur Phänomenologie der Intersubjektivität. Texte aus dem Nachlass. Erster Teil, 1905-1920, hg. von I. Kern, Den Haag 1973.

- (Hua XIV), Zur Phänomenologie der Intersubjektivität. Texte aus dem Nachlass. Zweiter Teil, 1921-1928, hg von I. Kern, Den Haag 1973.

- (Hua XV), Zur Phänomenologie der Intersubjektivität. Texte aus dem Nachlass. Dritter Teil, 1929-1935, hg. von I. Kern, Den Haag 1973.

Jensen, R. T., Motor intentionality and the case of Schneider, Phenomenology and the Cognitive Sciences 8, 2009, 371-388.

Laqueur, T. W., Auf den Leib geschrieben. Die Inszenierung der Geschlechter von der Antike bis Freud, Frankfurt a.M. 1992.

Lorenz, M., Leibhaftige Vergangenheit. Einführung in die Körpergeschichte, Tübingen 2000.

Lübke, V., CyberGender, Königstein 2005.

Maturana, H. \& Varela, F. J., Autopoiesis and Cognition: The Realization of the Living, Dordrecht 1980.

Meltzoff, A. N., Imitation and other minds. The ,like me' hypothesis, in: S. Hurley \& N. Chater (Hg.), Perspectives on Imitation: From Neuroscience to Social Science, Cambridge, MA 2005, 55-77.

Meltzoff, A. N. \& Moore, M. K., Imitation in newborn infants: exploring the range of gestures imitated and the underlying mechanisms, Developmental Psychology 25, 1989, 954-962.

- Explaining facial imitation: a theoretical model, Early Development and Parenting 6, 1997, 179-192.

Merleau-Ponty, M., Die Phänomenologie der Wahrnehmung, übers. von R. Böhm, Berlin/New York 1966.

- Vorlesungen I, Berlin 1972

- Das Sichtbare und das Unsichtbare, hg. von C. Lefort, übers. von R. Giuliani und B. Waldenfels, München, 2. Aufl., 1994.

- Keime der Vernunft. Vorlesungen an der Sorbonne 1949-1952, hg. von B. Waldenfels, München 1994.

- Das Auge und der Geist. Philosophische Essays, Hamburg 2003.

Nakamura, L., Cybertypes. Race, Ethnicity, and Identity on the Internet, New York 2002.

Nardi, B. A., My Life as a Night Elf Priest. An Anthropological Account of World of Warcraft, Ann Arbor 2010.

Plessner, H., Die Stufen des Organischen und der Mensch. Einleitung in die philosophische Anthropologie, Berlin 1965.

Rizzolatti, G., Fadiga, L., Gallese, V. \& Fogassi, L., Premotor cortex and the recognition of motor actions, Cognitive Brain Research 3, 1996, 131-141. 
Rizzolatti, G. \& Craighero, L., The mirror neuron system, Annual Review of Neuroscience 27, 2004, 169-192.

Roelcke, V., Krankheit und Kulturkritik. Psychiatrische Gesellschaftsdeutungen im bürgerlichen Zeitalter (1790-1914), Frankfurt a.M. 1999.

Sartre, J.-P., Das Sein und das Nichts, Reinbek 1993.

Staudigl, M., Racism: on the phenomenology of embodied desocialization, Continental Philosophical Review 45, 2012, 23-39.

Stoff, H., Diskurse und Erfahrungen. Ein Rückblick auf die Körpergeschichte der neunziger Jahre, 1999 14, 1999, 142-160.

Stoller, S., Wahrnehmung bei Merleau-Ponty, Frankfurt a.M. u.a. 1995.

Thompson, E., Mind in Life: Phenomenology, Biology and the Sciences of Mind, Cambridge, MA 2007.

Varela, F. J., Thompson, E. \& Rosch, E., The Embodied Mind. Cognitive Science and Human Experience, Cambridge, MA 1992

Waelhens, A. de, Une philosophie de l'ambiguité. L'existentialisme de Maurice Merleau-Ponty, Paris 1951.

Waldenfels, B., In den Netzen der Lebenswelt, Frankfurt a.M. 1985.

- Das leibliche Selbst. Vorlesungen zur Phänomenologie des Leibes, hg. von R. Giuliani, Frankfurt a.M. 2000.

Wehrle, M., Art. Intersubjektivität, in: H.-H. Gander (Hg.), HusserlLexikon, Darmstadt 2010, 158-164.

Zahavi, D., Husserls's Phenomenology, Stanford 2003. 